\title{
Genetic and Neurochemical Modulation of Prefrontal Cognitive Functions in Children
}

\author{
Adele Diamond, Ph.D. \\ Lisa Briand, B.S. \\ John Fossella, Ph.D. \\ Lorrie Gehlbach, Ph.D.
}

\begin{abstract}
Objective: The catechol $O$-methyltransferase (COMT) gene affects how long dopamine acts in the prefrontal cortex. The Methionine polymorphism, which results in a slower breakdown of prefrontal dopamine, is associated with better adult prefrontal cortex function. The authors investigated the relation between the COMT gene polymorphism and cognitive performance in children.
\end{abstract}

Method: Children were tested on cognitive tasks that depend on the dorsolateral prefrontal cortex and seem to be sensitive to the level of dopamine there (dots-mixed task), depend on that neural region but appear insensitive to its dopamine content (self-ordered pointing), and depend on other neural systems (recall memory and mental rotation). After data collection, cheek swabs were obtained from all children. DNA was extracted and genotyped for the COMT gene with polymerase chain reaction.
Results: Children who were homozygous for the Methionine polymorphism performed significantly better on the dotsmixed task but not on others.

Conclusions: The findings provide an existence proof that genotypic differences can relate to differences in cognitive performance in typically developing children. The authors achieved a level of specificity never previously attempted; the COMT polymorphism was found to be differentially related to performance on tasks linked to the same prefrontal region by whether cognitive requirements of the tasks were sensitive to the level of dopamine found. These results challenge accepted notions that since dopamine is important for some cognitive functions dependent on the prefrontal cortex, it is important for all. The differential sensitivity of distinct cognitive abilities to specific neurotransmitters may make possible targeted pharmacological interventions.
D dorsolateral prefrontal cortex. Reducing or blocking dopamine in the dorsolateral prefrontal cortex (Brodmann's areas 9, 46, and 9/46) produces deficits on tasks that are dependent on this region and that require working memory and inhibition, such as the delayed response task (1), which is comparable to the deficits produced by removing the dorsolateral prefrontal cortex altogether (2). Local injection of selective dopamine $\left(\mathrm{D}_{1}\right)$ antagonists into the dorsolateral prefrontal cortex impairs performance on such tasks in a precise dose-dependent manner $(3,4)$, leaving performance on control tasks unaffected. The concentration of extracellular dopamine in the dorsolateral prefrontal cortex (but not in other frontal regions) increases while monkeys are performing such tasks but not during performance of a control task (5). Dopamine strongly modulates the electrical activity of neurons in the prefrontal cortex $(6,7)$.

The self-ordered pointing task, which requires remembering stimuli already chosen and selecting new stimuli without repeating a choice, appears to be an exception to this general rule, according to the following:

1. Studies of normal brain function and of deficits seen after brain damage in humans and monkeys clearly show that success on the self-ordered pointing task requires use of the dorsolateral prefrontal cortex. Patients with damage to the dorsolateral prefrontal cortex fail the self-ordered pointing task (8-10), blood flow in the prefrontal cortex increases during performance of the self-ordered pointing task in normal adults (11), and rhesus macaques with lesions of the dorsolateral prefrontal cortex fail trials of a self-ordered pointing task variant (12). However, depleting the prefrontal cortex of dopamine does not impair performance on the self-ordered pointing task (13), although the same depletion does impair performance of the delayed response task, and lesions of the prefrontal cortex impair performance on both tasks.

2. Similarly, children treated early and continuously for phenylketonuria, with plasma phenylalanine levels of 6-10 mg/dl, are hypothesized to have lower levels of dopamine in the prefrontal cortex (a hypothesis supported in an animal model [14]) and to perform normally on the self-ordered pointing task, although the same children have been found to be impaired on a host of tasks dependent on the dorsolateral prefrontal cortex that require working memory and inhibition (15). 
It is not clear why self-ordered pointing appears insensitive to the level of dopamine in the dorsolateral prefrontal cortex, although that task depends on functioning of this region. One possibility is that while it requires holding information in mind and manipulating it, it may not require inhibition; different cognitive functions that are dependent on the dorsolateral prefrontal cortex may be differentially sensitive to dopamine activity in that region.

Dopamine projection to the prefrontal cortex is unusual in several ways. Dopamine neurons that project to the prefrontal cortex are distinct from dopamine neurons that project to the striatum and even from those that project to the nucleus accumbens or the anterior cingulate (16). Their baseline rate of firing is higher, as is their rate of dopamine turnover $(17,18)$. They contain much less dopamine transporter, and the dopamine transporter there is poorly situated (at a distance from synaptic release sites [19, unpublished work by Sanchez and Cavada]). These distinguishing characteristics are important because they provide a mechanism by which the effect of genetic polymorphisms affecting dopamine can have different, potentially greater, effect on the dopamine system in the prefrontal cortex than on other dopamine systems.

Those higher firing and dopamine turnover rates make the prefrontal cortex more sensitive to modest reductions in the level of tyrosine (20). Apparently because of that, working memory and inhibition functions that depend on the dorsolateral prefrontal cortex (but not cognitive functions dependent on other neural systems, such as the parietal cortex or the medial temporal lobe) are impaired in children treated for phenylketonuria whose serum phenylalanine levels are elevated enough to result in a modest reduction in tyrosine levels reaching the brain $(14,15,21)$.

The catechol $O$-methyltransferase (COMT) gene, mapped to human chromosome 22q11 $(22,23)$, codes for the COMT enzyme, which is important for the degradation of dopamine released into synapses. The COMT enzyme catalyzes the transfer of a methyl group to a hydroxyl group on catecholamines such as dopamine and norepinephrine $(24,25)$.

A functional missense mutation of the COMT gene (a single base-pair substitution: CGTG to CATG) results in a substitution of Methionine (Met) for Valine (Val) at codon 108/158: AGVKD versus AGMKD (26). This Val-to-Met polymorphism has a significant effect on COMT enzymatic activity. The enzyme containing Met at that position is four times less active and breaks down dopamine more slowly (27), allowing dopamine to remain active in and around synaptic clefts longer.

This polymorphism has a regionally greater selective effect on the prefrontal cortex than on other neural regions, such as the striatum. That is because the prefrontal cortex is particularly dependent on the COMT pathway to terminate the action of synaptic dopamine, given the prefrontal cortex's paucity of dopamine transporter protein (19).
Dopamine transporter protein is critical for dopamine reuptake. With less reuptake by the dopamine transporter, the prefrontal cortex is more dependent on secondary mechanisms, such as COMT degradation, to inactivate extracellular dopamine.

The striatum, in contrast, is less dependent on COMT degradation because of its abundant and well-situated dopamine transporter (28). COMT methylation accounts for $\leq 15 \%$ of total dopamine turnover in the striatum and nucleus accumbens but $>60 \%$ in the prefrontal cortex (29).

The Met polymorphism of the COMT gene, which results in slower dopamine catabolism, is associated with better function of the prefrontal cortex in adults (30). Adults homozygous for the Met polymorphism perform better on the Wisconsin Card Sorting Test $(30,31)$ and need less neuronal activity in the prefrontal cortex to achieve a given level of performance on the N-back test than adults with $2 \mathrm{Val}$ alleles (found in three separate samples [30]). These effects are specific to the functions of the prefrontal cortex. The Met versus Val polymorphism is uncorrelated with IQ or other non-prefrontal-cortex functions (30). The Wisconsin Card Sorting and N-back tests require working memory and inhibition and recruit the dorsolateral prefrontal cortex (32-34).

Such findings are consistent with those obtained when COMT's enzymatic activity is inhibited, which results in slower clearance of released dopamine (as does the Met polymorphism). Administering tolcapone (a selective COMT inhibitor) to patients with depleted dopamine levels improves their performance on working memory and inhibition tasks that are dependent on the prefrontal cortex (35) but does not improve parkinsonian motor symptoms associated with striatal function (36).

It is not entirely clear why the slower COMT works, the more efficiently the prefrontal cortex functions. Certainly, too much dopamine impairs function of the prefrontal cortex, as does too little (37). The detrimental effects of too much dopamine occur on primary dendritic stems, far from the terminals, whereas dopamine $\mathrm{D}_{1}$ receptors are located closer, only a short distance from the terminals. COMT is localized near the terminals. Thus, one possibility is that if COMT acts more slowly, more released dopamine might reach dopamine receptors.

\section{Method}

We tested typically developing children in our laboratory on a task that requires working memory and inhibition and depends on function of the dorsolateral prefrontal cortex (the dots-mixed task) $(14,38)$, the self-ordered pointing task (which requires working memory but probably not inhibition and depends on the dorsolateral prefrontal cortex), recall memory (dependent on the medial temporal lobe), and body-image mental rotation (dependent on the posterior parietal cortex).

The evidence linking recall memory to the medial temporal lobe is overwhelming, and impaired recall is the prototypic impairment in temporal lobe amnesia (39). The "hands" mental rotation task used here, as developed by Coslett (40), building on 
FIGURE 1. Stimuli in the Dots-Mixed Task ${ }^{\mathrm{a}}$

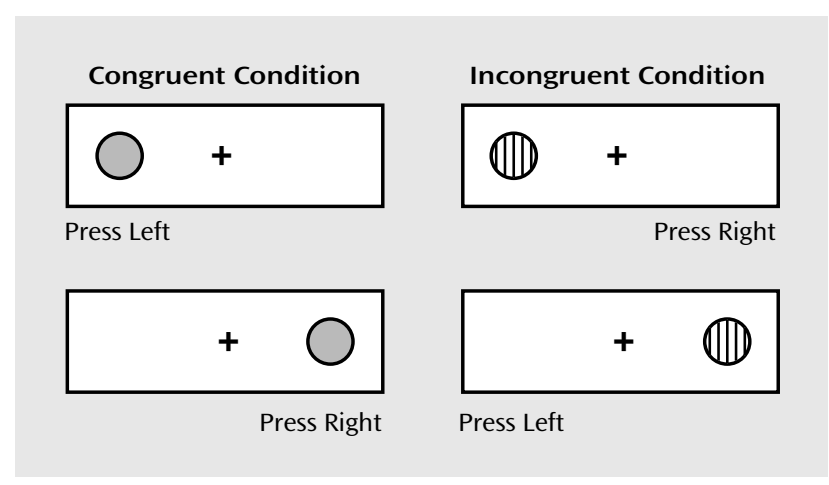

${ }^{\mathrm{a}}$ In the congruent condition, the correct response is to press the dot on the same side as the stimulus. In the incongruent condition, the response and stimulus are on opposite sides. In the mixed condition, equal numbers of congruent and incongruent trials are randomly intermixed.

the work of others (41), is sensitive to parietal cortex damage (40, 42), and performance of similar tasks increases parietal cortex activation (43). Diverse mental rotation measures have produced consistently robust parietal activation and shown marked sensitivity to parietal damage in both adults (44-46) and children (47).

The children had been recruited through local school districts by letters to parents sent home with the children and by parents hearing about us through the Web or word of mouth and contacting us. Letters sent home were accompanied by our telephone number and by reply cards that parents returned if they wanted us to call to provide more information. Preliminary information provided over the telephone was supplemented when the family arrived at our laboratory. Screening was conducted over the telephone and confirmed at the start of the testing session. Children who had any known learning disability, serious health problem, developmental delay, or complications at birth; were not fluent in English; had been born prematurely; or were taking psychotropic medication were not included in the study. Testing was conducted in quiet child-friendly rooms. Each child received a small present of his or her choice for participating.

Later, we returned to the children's parents to ask if we might obtain a cheek swab for DNA testing. Our predictions were as follows:

1. The COMT polymorphism should correlate with performance on the dots-mixed task.

2. Specifically, the children homozygous for the Met allele should perform better on the dots-mixed task than those with the Val-Val genotype.

3 . This same genetic variation should not correlate with performance on the self-ordered pointing task, since performance on that is insensitive to dopamine levels in the dorsolateral prefrontal cortex.

4. This genetic variation also should not correlate with recall memory or mental rotation performance since those mental operations do not depend on the dorsolateral prefrontal cortex.

Separate informed consent was obtained for the cheek (buccal) swabs. For both the behavioral testing and the DNA analyses from the buccal swabs, written informed consent was obtained.

The directional Stroop task was developed to vary demands independently on working memory and inhibition with a task appropriate for preschoolers through adults and for functional magnetic resonance imaging (14). The dots-mixed condition (Figure 1) requires that participants remember two rules and inhibit the
FIGURE 2. A Child Responding in a Six-Item Trial During a Computerized Self-Ordered Pointing Task ${ }^{\mathrm{a}}$

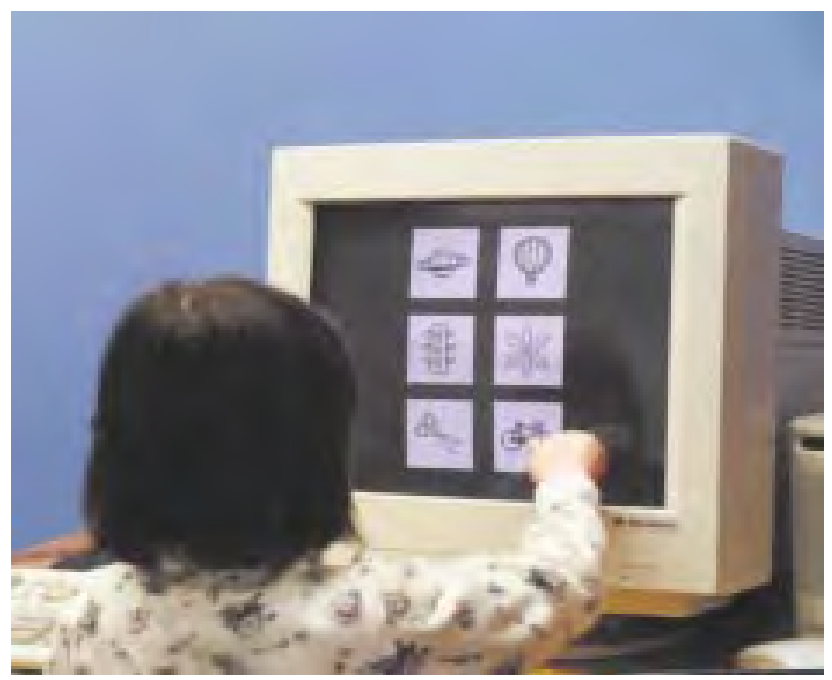

a Each time an item is touched, the screen is refreshed, and the items reappear in the same grid. However, the location of any item in the grid will have changed, so previous responses must be encoded by the appearance of the stimulus, not by location. Choosing the same spatial location repeatedly would not result in a good score.

tendency to respond on the same side as the stimulus on one-half of the trials.

The impulse to respond on the same side as the stimulus is well documented. People are slower and less accurate when responding on the side opposite a stimulus than when the stimulus and correct response are on the same side $(48,49)$ ("the Simon effect"). Indeed, when monkeys must point away from a stimulus, the neuronal population vector in the motor cortex initially points toward the stimulus and only then shifts to the required direction, indicating a prepotent tendency to respond toward a stimulus; doing otherwise requires its inhibition (50).

Using a self-held button box with a button for the right and left hands, participants indicated the correct response for each stimulus as it appeared on a computer screen. One-half of the participants were told to press the button on the same side as the stimulus when it was gray and on the opposite side when it was striped; one-half were told the opposite. After practice, the participants performed a block of 20 trials in the congruent (same-side) condition, a block of 20 incongruent (opposite-side) trials, and then 20 mixed trials. The interstimulus interval was $500 \mathrm{msec}$; stimuli were presented for $750 \mathrm{msec}$ for those $\geq 7$ years of age and for 2500 msec for younger children. Feedback was provided during training but never during performance of the test. Responses of $\leq 200$ msec were not calculated in the percentage of correct responses or reaction time since those were too rapid to be in response to the stimulus.

Age norms on the directional Stroop task, including the dotsmixed variant, have been established (unpublished study by Davidson et al.). The dependence of performance of the dotsmixed task on the dorsolateral prefrontal cortex has been revealed by an increase in activation there compared to performance on the dots-congruent task (only gray dots), the dotsincongruent variant (only striped dots), or the arrows-mixed task (which requires inhibition but little working memory) (38, unpublished work by Diamond et al.).

In the Petrides-Milner self-ordered pointing task (8) we use, participants perform six blocks of trials, two each for the set of six line drawings, eight line drawings, and 12 abstract designs, each 


\begin{tabular}{|c|c|c|c|c|c|c|}
\hline \multirow[t]{2}{*}{ Characteristic } & \multicolumn{2}{|c|}{ Met-Met $(\mathrm{N}=9)$} & \multicolumn{2}{|c|}{ Val-Met $(\mathrm{N}=16)$} & \multicolumn{2}{|c|}{ Val-Val $(\mathrm{N}=14)$} \\
\hline & Mean & Range & Mean & Range & Mean & Range \\
\hline \multirow[t]{2}{*}{ Age (years) } & 10.0 & $8.0-12.8$ & 8.5 & $6.8-11.5$ & 10.1 & $8.2-14.6$ \\
\hline & $\mathrm{N}$ & $\%$ & $\mathrm{~N}$ & $\%$ & $N$ & $\%$ \\
\hline $\begin{array}{l}\text { Female } \\
\text { gender } \\
\text { Ethnicity }\end{array}$ & 5 & 56 & 5 & 31 & 6 & 43 \\
\hline Caucasian & 9 & 100 & 13 & 81 & 13 & 92 \\
\hline Multiethnic & 0 & 0 & 2 & 13 & 1 & 8 \\
\hline \multirow[t]{2}{*}{ Asian } & 0 & 0 & 1 & 6 & 0 & 0 \\
\hline & Mean & SD & Mean & SD & Mean & SD \\
\hline \multicolumn{7}{|l|}{$\begin{array}{l}\text { Parental } \\
\text { education } \\
\text { (years) }\end{array}$} \\
\hline Mother & 16.6 & 0.9 & 17.1 & 1.6 & 17.4 & 1.9 \\
\hline Father & 17.4 & 1.7 & 16.0 & 2.6 & 16.0 & 2.4 \\
\hline
\end{tabular}

presented in a rectangular grid (Figure 2). Participants are to touch one stimulus at a time, in any order, without repeating a choice, so that all stimuli are touched but each only once. After each response, the computer screen refreshes, and the same stimuli are presented in the same grid, but the locations of individual stimuli have changed. Thus, previous responses must be encoded by stimulus appearance-not location. Simply touching the same position does not result in a good score. Each stimulus grid is presented until a response is recorded. Each block of trials is the same length as the number of stimuli in that set (six, eight, or 10). Feedback is provided during training but never during testing. This task has been successfully used with children by ourselves and others $(15,51,52)$.

To assess recall memory, we asked the children to study a set of six and a set of eight single-item line drawings, telling the children that they would later be asked to recall those items. At the end of the testing session (an hour later), the children were asked to name or describe all of the items they could remember from the 14 shown earlier. To assess mental rotation, eight photographs were used (four each of a right and left hand, two palm up and two palm down), first shown at $0^{\circ}$ rotation and then all rotated $180^{\circ}$ $(40,42)$. For each of the 16 trials, the children indicated whether the photograph showed a right or left hand. Their own hands were concealed under a cloth with a turtle sticker on one arm and a bunny sticker on the other; they could respond by saying "right/ left hand" or "turtle/bunny hand."

Buccal swabs, obtained with a cytology brush (MasterAMP Buccal Swab DNA Extraction Kit, Epicentre Inc., Madison, Wis.), with the consent of parent and child (and approval of the institutional review board of the University of Massachusetts Medical School) provided the tissue from which DNA was extracted. Swabs were air-dried to discourage bacterial growth, and samples were purified with Quick-Extract DNA Extraction Solution (Epicentre Inc., Madison, Wis.). Yields, as determined spectrophotometrically by absorbance at $260 \mathrm{~nm}$, ranged from 0.5 to $3 \mu \mathrm{g}$ of DNA from each buccal sample. Taq polymerase, polymerase chain reaction (PCR) buffer, and deoxynucleotide triphosphates were obtained from QIAGEN Genomics, Inc. (Bothell, Wash.), and used at recommended concentrations for a $20 \mu \mathrm{l}$ PCR reaction. For genotyping of the COMT Val-to-Met polymorphism at codon 158, forward 5'-TCACCATCGAGATCAACCCC-3' and reverse $5^{\prime}$ GAACGTGGTGTGAACACCTG-3' primers were used (53). For genotyping of the COMT Val-to-Met polymorphism at codon 108, forward 5'-ACTGTGGCTACTCAGCTGTG-3' and reverse 5' CCTTTTTCCAGGTCTGACAA-3' primers were used (54). PCR re- actions were optimized with a touchdown PCR cycling regimen and the addition of dimethyl sulfoxide ( $10 \%$ final volume to volume) and performed on a PTC-100 Programmable Thermal Controller (MJ Research, Waltham, Mass.) outfitted with a heated lid for oil-free amplifications. Restriction fragment-length polymorphisms were detected by NlaIII restriction digests and subsequent gel electrophoresis in 3\% Metaphor agarose followed by staining in ethidium bromide.

\section{Results}

We report data here on 39 children, most under the age of 12 (mean age=9 years). The Met polymorphism of COMT is common, with an incidence of roughly $40 \%-50 \%$ in Europe and North America (55), as was reflected in our study group (Table 1). Because the number of subjects genotyped per group was not large and because the scores for Val-Val children were not evenly distributed, we used nonparametric tests: Kruskal-Wallis tests for comparing all three groups and Wilcoxon Mann-Whitney tests for pairwise comparisons (StatXact, Cytel Software, Inc., Cambridge, Mass.). (On the dots-mixed task, all Val-Val children performed worse than, or close to, the norm for their age, except for one child who performed exceptionally well.)

Our predictions were confirmed. The children with the Met-Met genotype performed significantly better on the dots-mixed task than the Val-Val children (Wilcoxon $\mathrm{t}=$ 126.0, p<0.01) (Figure 3). The heterozygous children performed intermediately well, which was not significantly different from that of the other groups. These results (with a different cognitive assay) replicate, for the first time in children, results obtained with adults $(30,31)$. There was no relation between versions of the COMT gene and performance on the nonprefrontal cognitive measures (recall memory-Met-Met versus Val-Val: Wilcoxon $\mathrm{t}=50.0, \mathrm{p}=$ 0.43; mental rotation-Met-Met versus Val-Val: Wilcoxon $\mathrm{t}=61.8, \mathrm{p}=0.43$ ). The Kruskal-Wallis values for comparisons of the three subject groups were $\mathrm{t}=3.6, \mathrm{p}<0.05$, for the dotsmixed task; $\mathrm{t}=1.6, \mathrm{p}=0.45$, for self-ordered pointing; $\mathrm{t}=0.8$, $\mathrm{p}=0.69$ for recall memory; and $\mathrm{t}=1.8, \mathrm{p}=0.41$, for mental rotation. There was a sex difference on one of the tasks (mental rotation: Wilcoxon $\mathrm{t}=166.0, \mathrm{p}<0.005)$. Controlling for that did not alter the lack of any difference by COMT genotype on performance of the task.

No difference was found between the performance of the two homozygous groups of children on the self-ordered pointing task $(\mathrm{t}=64.0, \mathrm{p}=0.47$ ) (Figure 3 ) nor between the heterozygotes and either homozygous group. This represents a greater level of specificity than had been obtained before. It also provides a third converging line of evidence that although successful performance on the self-ordered pointing task (a measure of working memory) relies on the dorsolateral prefrontal cortex and although dopamine is an important neurotransmitter in the dorsolateral prefrontal cortex upon which many of the cognitive functions that require the dorsolateral prefrontal cortex 
depend, performance on the self-ordered pointing task (unlike many other cognitive measures that require working memory plus inhibition and depend on the dorsolateral prefrontal cortex) is not affected by changes in the level of dopamine in the dorsolateral prefrontal cortex.

This represents a greater level of specificity than had been obtained before, although successful performance on the self-ordered pointing task (a measure of working memory) relies on the dorsolateral prefrontal cortex and dopamine is an important neurotransmitter in this region upon which many of the cognitive functions that require this region depend. It also provides a third converging line of evidence that performance on the self-ordered pointing task (unlike many other cognitive measures that require working memory and inhibition and depend on the dorsolateral prefrontal cortex) is not affected by changes in the level of dopamine in the dorsolateral prefrontal cortex.

\section{Discussion}

In sum, the Met polymorphism of the COMT gene has a selective effect on the prefrontal cortex's dopamine system because of the greater dependence of the prefrontal cortex on the COMT pathway to clear extracellular dopamine. Tasks that require working memory and inhibition are sensitive to the dopamine level in the dorsolateral prefrontal cortex. The self-ordered pointing task that requires working memory but perhaps not inhibition is not, although it, too, depends on the dorsolateral prefrontal cortex. In most children, the slower-acting Met-Met polymorphism (which allows released dopamine to remain active longer in the prefrontal cortex) resulted in better performance than the Val-Val COMT genotype on a task requiring working memory and inhibition (the dots-mixed task) but not on the self-ordered pointing task. This is consistent with the hypothesized effect of the COMT gene polymorphism and with independent evidence that self-ordered pointing is insensitive to dopamine levels in the prefrontal cortex. These results appear to challenge accepted notions that since dopamine is important for some cognitive functions that depend on the dorsolateral prefrontal cortex, it is important for all cognitive functions that depend on that neural region. The differential sensitivity of distinct cognitive abilities to specific neurotransmitters may make possible targeted pharmacological interventions.

It is possible, though unlikely, that the COMT genotype (i.e., the level of dopamine in the prefrontal cortex) was related to performance on the dots-mixed task but not the self-ordered pointing, recall memory, or mental rotation task. This may be because for the latter three, the presentation rate of the stimulus was determined by the participant, whereas for dots-mixed stimuli, presentation time was pre-set and brief. It is likely that reducing the level of dopamine in the prefrontal cortex impairs functioning less than does ablation of the prefrontal cortex, so that while performance on the self-ordered pointing task is im-
FIGURE 3. Performance of Healthy Children on Cognitive Measures, by COMT Genotype ${ }^{a}$

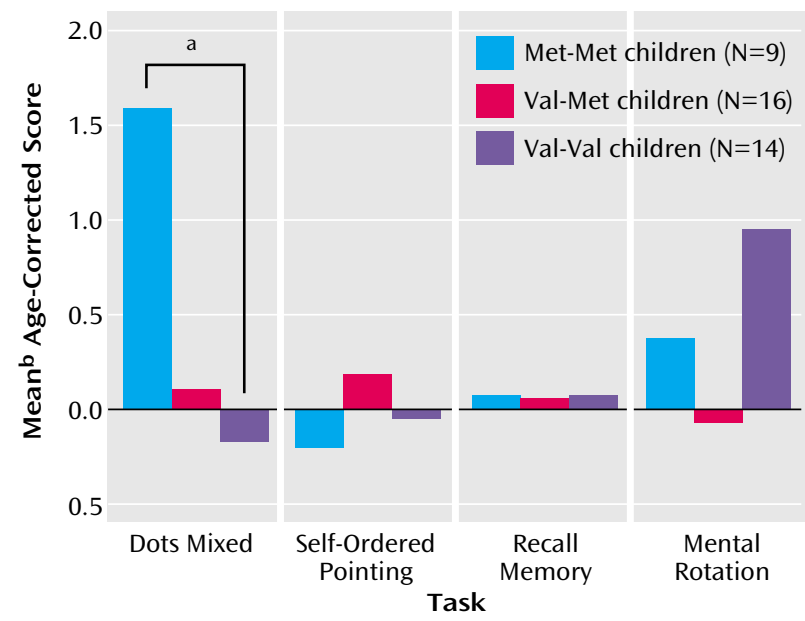

a To calculate each child's age-adjusted score, we subtracted the mean score for children of that child's age from the child's score. To control for the effect of age, mean differences in scores by age were used. For each task, the mean percentage of correct responses for the subject's age in years was subtracted from the subject's percentage of correct responses, yielding an age difference score. This partialled out any effect of age. Gender was not significantly related to performance on any cognitive task except mental rotation. The children with the Met-Met genotype performed significantly better than the children with the Val-Val genotype on the dots-mixed task, which requires holding two higher-order rules in mind and switching between inhibiting a prepotent response and making that response and is sensitive to the level of dopamine in the prefrontal cortex. All three groups performed comparably on the dots-congruent trial block (with the dot always pressed on the same side as the stimulus) and the dots-incongruent trial block (with the dot always pressed on the opposite side), as expected. Those conditions require holding only one higher-order rule in mind, and either no inhibition (dots-congruent) or steady-state inhibition (always inhibit pressing the dot on same side as the stimulus). All groups performed comparably on self-ordered pointing, which is not sensitive to the level of dopamine in the prefrontal cortex. No difference between Met-Met and Val-Val children was found on the six-, eight-, or 12 -item self-ordered pointing trials or on all combined. Performance on the eight-item trials is shown here since that intermediate level of difficulty was definitely not susceptible to ceiling or floor effects. All groups also performed comparably on our two control tasks: recall memory and mental rotation.

b SDs were the following for the Met-Met, Val-Met, and Val-Val genotypes, respectively: dots-mixed task-2.01, 3.37, and 2.74; self-ordered pointing task-0.99, 1.06, and 1.05; recall memory task$0.07,0.15$, and 0.13 ; mental rotation-2.98, 2.44, and 1.92 .

paired by cortex lesions, it is not impaired by a reduced dopamine level in the dorsolateral prefrontal cortex. That would mean that self-ordered pointing is more robust to a less severe deficit of functioning in the dorsolateral prefrontal cortex than is the dots-mixed task, which is impaired even by reducing dopamine in the dorsolateral prefrontal cortex. The dots-mixed task may be a more sensitive measure in general than self-ordered pointing, since variance in performance of the dots-mixed task is greater than that for self-ordered pointing.

It is not simply that any sensitive task detects a difference in performance by the COMT genotype since between-subject variation in the performance of mental rotation was 
comparable to that for the dots-mixed task. However, no difference in performance by the COMT genotype was detected for mental rotation. Differences in motivation or arousal cannot account for our results because the 12-item portion of the self-ordered pointing task was the most difficult portion of any of the tests, but no group differences were found on either the six-item portion, which was the easiest, or on the eight-item portion. Had low arousal or motivation been an issue, group differences would have been expected the more difficult the measure; such was not found.

These findings provide an existence proof that differences in genotype can relate to differences in cognitive performance in typically developing children. Even among a small group of such children, differences in the COMT phenotype correlated significantly and in the predicted direction, with differences in performance on a task dependent on the prefrontal cortex and its dopamine content. Gene association studies that are focused on wellstudied candidate genes do not require the large group sizes needed for exploratory studies. For example, in a group of only 14 normal older adults, differences in memory performance correlated with the presence or absence of one copy of the APOE-4 allele (56).

Analyses of genes, such as COMT, that affect neurotransmitter systems provide a window into the neurochemical modulation of the working memory and inhibitory abilities that are dependent on the prefrontal cortex. Those abilities are of critical importance. The ability to inhibit attention to distractors makes possible selective and sustained attention. The ability to inhibit a prepotent response helps make flexibility and change possible. Inhibition, thus, allows us a measure of control over our attention and our actions. The ability to hold information in mind enables us to remember our plans or others' instructions, consider alternatives, and relate one idea or datum to another.

A better understanding of genetic modulation of these cognitive abilities should provide guidance for protecting children against developing — or for mitigating the severity of-deficits in functions dependent on the prefrontal cortex that are implicated in so many disorders, including attention deficit hyperactivity disorder $(57,58)$, obsessivecompulsive disorder (59), addiction (60), schizophrenia (61), and depression $(62,63)$.

Presented in part at the annual meeting of the American Association for the Advancement of Science, Boston, Feb. 14-19, 2002. Received June 26, 2002; revisions received Jan. 28, 2003, and April 30, 2003; accepted May 13, 2003. From the Center for Developmental Cognitive Neuroscience, Eunice Kennedy Shriver Center Campus, University of Massachusetts Medical School; and the Sackler Institute, Weill Medical College, Cornell University, New York. Address reprint requests to Dr. Diamond, Center for Developmental Cognitive Neuroscience, Eunice Kennedy Shriver Center Campus, University of Massachusetts Medical School, 200 Trapelo Rd., Waltham, MA 02452; adele.diamond@umassmed.edu (e-mail).

Supported by a grant (HD-35453) from the National Institute of Child Health and Development.
The authors thank the parents and children who participated in this study, Donald Druin for consultation concerning the handling of DNA, Jean Mack for contacting parents and arranging appointments, and Sylvia Baedorf, Kim Dilda, and Sarah Groff for assistance in obtaining the buccal swabs.

\section{References}

1. Diamond A, Goldman-Rakic PS: Comparison of human infants and rhesus monkeys on Piaget's A-not-B task: evidence for dependence on dorsolateral prefrontal cortex. Exp Brain Res 1989; 74:24-40

2. Brozoski TJ, Brown RM, Rosvold HE, Goldman PS: Cognitive deficit caused by regional depletion of dopamine in prefrontal cortex of rhesus monkey. Science 1979; 205:929-932

3. Sawaguchi T, Goldman-Rakic PS: $D_{1}$ dopamine receptors in prefrontal cortex: involvement in working memory. Science 1991; 251:947-950

4. Seamans JK, Floresco SB, Phillips AG: $D_{1}$ receptor modulation of hippocampal-prefrontal cortical circuits integrating spatial memory with executive functions in the rat. J Neurosci 1998; 18:1613-1621

5. Watanabe M, Kodama T, Hikosaka K: Increase of extracellular dopamine in primate prefrontal cortex during a working memory task. J Neurophysiol 1997; 78:2795-2798

6. Williams GV, Goldman-Rakic PS: Modulation of memory fields by dopamine $D_{1}$ receptors in prefrontal cortex. Nature 1995; 376:572-575

7. Yang CR, Seamans JK: Dopamine $\mathrm{D}_{1}$ receptor actions in layers $\mathrm{V}-\mathrm{V} 1$ rat prefrontal cortex neurons in vitro: modulation of dendritic-somatic signal integration. J Neurosci 1996; 16:19221935

8. Petrides M, Milner B: Deficits on subject-ordered tasks after frontal- and temporal-lobe lesions in man. Neuropsychologia 1982; 20:249-262

9. Wiegersma S, Van der Scheer E, Hijman R: Subjective ordering, short-term memory, and the frontal lobes. Neuropsychologia 1990; 28:95-98

10. Milner B, Corsi P, Leonard G: Frontal-lobe contribution to recency judgments. Neuropsychologia 1991; 29:601-618

11. Petrides M, Alivisatos B, Meyer E, Evans AC: Functional activation of the human frontal cortex during performance of verbal working memory tasks. Proc Natl Acad Sci USA 1993; 90:878882

12. Petrides M: Impairments on nonspatial self-ordered and externally ordered working memory tasks after lesions of the middorsal part of the lateral frontal cortex in the monkey. J Neurosci 1995; 15:359-375

13. Collins P, Roberts AC, Dias R, Everitt BJ, Robbins TW: Perseveration and strategy in a novel spatial self-ordered task for nonhuman primates: effect of excitotoxic lesions and dopamine depletions of the prefrontal cortex. J Cogn Neurosci 1998; 10: 332-354

14. Diamond A: A model system for studying the role of dopamine in prefrontal cortex during early development in humans: early and continuously treated phenylketonuria, in Handbook of Developmental Cognitive Neuroscience. Edited by Nelson C, Luciana M. Cambridge, Mass, MIT Press, 2001, pp 433-472

15. Diamond A, Prevor M, Callender G, Druin DP: Prefrontal cortex cognitive deficits in children treated early and continuously for PKU. Monogr Soc Res Child Dev 1997; 62(serial number 252):1207

16. Carr DB, Sesack SR: Projections from the rat prefrontal cortex to the ventral tegmental area: target specificity in the synaptic associations with mesoaccumbens and mesocortical neurons. J Neurosci 2000; 20:3864-3873 
17. Thierry AM, Tassin JP, Blanc G, Stinus L, Scatton B, Glowinski J: Discovery of the mesocortical dopaminergic system: some pharmacological and functional characteristics. Adv Biomed Psychopharmacol 1977; 16:5-12

18. Tam SY, Roth RH: Mesoprefrontal dopaminergic neurons: can tyrosine availability influence their functions? Biochem Pharmacol 1997; 53:441-453

19. Sesack SR, Hawrylak VA, Matus C, Guido MA, Levey AI: Dopamine axon varicosities in the prelimbic division of the rat prefrontal cortex exhibit sparse immunoreactivity for the dopamine transporter. J Neurosci 1998; 18:2697-2708

20. Bradberry CW, Karasic DH, Deutsch AY, Roth RH: Regionallyspecific alterations in mesotelencephalic dopamine synthesis in diabetic rats: associations with precursor tyrosine. J Neural Transm 1989; 78:221-229

21. Luciana M, Sullivan J, Nelson C: Associations between phenylalanine-to-tyrosine ratios and performance on tests of neurosychological function in adolescents treated early and continuously for phenylketonuria. Child Dev 2001; 72:1637-1652

22. Grossman MH, Emanuel BS, Budaf ML: Chromosomal mapping of the human catechol-O-methyltransferase gene to $22 \mathrm{q} 11.1$ q11.2. Genomics 1992; 12:822-825

23. Tenhunen J, Salminen M, Lundstrom K, Kiviluoto T, Savolainen $\mathrm{R}$, Ulmanen I: Genomic organization of the human catechol Omethyltransferase gene and its expression from two distinct promotors. Eur J Biochem 1994; 223:1049-1059

24. Napolitano A, Cesura AM, Da Prada M: The role of monoamine oxidase and catechol O-methyltransferase in dopaminergic neurotransmission. J Neural Transm 1995; 45:35-45

25. Weinshilboum RM, Otterness DM, Szumlanski CL: Methylation pharmacogenetics: catechol O-methyltransferase, thiopurine methyltransferase, and histamine $\mathrm{N}$-methyltransferase. Annu Rev Pharmacol Toxicol 1999; 39:19-52

26. Lachman HM, Papolos DF, Saito T, Yu YM, Szumlanski CL, Weinshilboum RM: Human catechol-O-methyltransferase pharmacogenetics: description of a functional polymorphism and its potential application to neuropsychiatric disorders. Pharmacogenetics 1996; 6:243-250

27. Lotta T, Vidgren J, Tilgmann C, Ulmanen I, Melen K, Julkunen I, Taskinen J: Kinetics of human soluble and membrane-bound catechol O-methyltransferase: a revised mechanism and description of the thermolabile variant of the enzyme. Biochemistry 1995; 34:4204-4210

28. Garris PA, Wrightman RM: Different kinetics govern dopaminergic transmission in the amygdala, prefrontal cortex, and striatum: an in vivo voltametric study. J Neurosci 1994; 14:442450

29. Karoum F, Chrapusta SJ, Egan MF: 3-Methoxytryramine is the major metabolite of released dopamine in the rat frontal cortex: reassessment of the effects of antipsychotics on the dynamics of dopamine release and metabolism in the frontal cortex, nucleus accumbens, and striatum by a simple two pool model. J Neurochem 1994; 63:972-979

30. Egan MF, Goldberg TE, Kolachana BS, Callicott JH, Mazzanti CM, Straub RE, Goldman D, Weinberger DR: Effect of COMT Val108/ 158 Met genotype on frontal lobe function and risk for schizophrenia. Proc Natl Acad Sci USA 2001; 98:6917-6922

31. Malhotra AK, Kestler LJ, Mazzanti C, Bates JA, Goldberg T, Goldman D: A functional polymorphism in the COMT gene and performance on a test of prefrontal cognition. Am J Psychiatry 2002; 159:652-654

32. Smith EE, Jonides J: Storage and executive processes in the frontal lobes. Science 1999; 283:1657-1661

33. Stuss DT, Levine B, Alexander MP, Hong J, Palumbo C, Hamer L, Murphy KJ, Izukawa D: Wisconsin Card Sorting Test performance in patients with focal frontal and posterior brain dam- age: effects of lesion location and test structure on separable cognitive processes. Neuropsychologia 2000; 38:388-402

34. Monchi O, Petrides M, Petre V, Worsley K, Dagher A: Wisconsin Card Sorting revisited: distinct neural circuits participating in different stages of the task identified by event-related functional magnetic resonance imaging. J Neurosci 2001; 21:77337741

35. Gasparini M, Fabrizio E, Bonifati V, Meco G: Cognitive improvement during Tolcapone treatment in Parkinson's disease. J Neural Transm 1997; 104:887-894

36. Chong DJ, Suchowersky O, Szumlanski C, Weinshilboum RM, Brant R, Campbell NR: The relationship between COMT genotype and the clinical effectiveness of tolcapone, a COMT inhibitor, in patients with Parkinson's disease. Clin Neuropharmacol 2000; 23:143-148

37. Arnsten AF: Catecholamine regulation of the prefrontal cortex. J Psychopharmacol 1997; 11:151-162

38. Diamond A, O'Craven KM, Savoy RLI: Dorsolateral prefrontal cortex contributions to working memory and inhibition as revealed by fMRI. Abstracts of the Society for Neuroscience 1998; 24:1251

39. Squire LR, Shimamura AP: Characterizing amnesic patients for neuro-behavioral study. Behav Neurosci 1986; 100:866-877

40. Coslett HB: Evidence for a disturbance of the body schema in neglect. Brain Cogn 1998; 37:527-544

41. Cooper LA, Shepard RN: Mental transformations in the identification of left and right hands. J Exp Psychol Hum Percept Perform 1975; 104:48-56

42. Schwoebel J, Friedman R, Duda N, Coslett HB: Pain and the body schema: evidence for peripheral effects on mental representations of movement. Brain 2001; 124:2098-2104

43. Bonda E, Petrides M, Frey S, Evans A: Neural correlates of mental transformations of the body-in-space. Proc Natl Acad Sci USA 1995; 92:11180-11184

44. Alivisatos B, Petrides M: Functional activation of the human brain during mental rotation. Neuropsychologia 1997; 35: 111-118

45. Cohen MS, Kosslyn SM, Breiter HC, DiGirolamo GJ, Thompson WL, Anderson AK, Bookheimer SY, Rosen BR, Belliveau JW: Changes in cortical activity during mental rotation: a mapping study using functional MRI. Brain 1996; 119:89-100

46. Newcombe F, Ratcliff G: Disorders of visuo-spatial analysis, in Handbook of Neuropsychology. Edited by Boller F, Grafman J. Amsterdam, Elsevier, 1989, pp 333-356

47. Booth JR, MacWhinney B, Thulborn KR, Sacco K, Voyvodic JT, Feldman HM: Developmental and lesion effects in brain activation during sentence comprehension and mental rotation. Dev Neuropsychol 2000; 18:139-169

48. Simon JR, Acosta E, Mewaldt SP, Speidel CR: The effect of an ir relevant directional cue on choice reaction time: duration of the phenomenon and its relation to stages of processing. Percept Psychophys 1976; 19:16-22

49. Lu CH, Proctor RW: The influence of irrelevant location information on performance: a review of the Simon and spatial Stroop effects. Psychon Bull Rev 1995; 2:174-207

50. Georgopoulos AP, Lurito JT, Petrides M, Schwartz AB, Massey JT: Mental rotation of the neuronal population vector. Science 1989; 243:234-236

51. Archibald SJ, Kerns KA: Identification and description of new tests of executive functioning in children. Child Neuropsychol 1999; 5:115-129

52. Smith ML, Klim P, Hanley WB: Executive function in schoolaged children with phenylketonuria. J Dev Phys Disabil 2000; 12:317-332

53. Barr CL, Wigg K, Malone M, Schachar R, Tannock R, Roberts W, Kennedy JL: Linkage study of catechol-O-methyltransferase 
and attention-deficit hyperactivity disorder. Am J Med Genet 1999; 15:710-713

54. Daniels JK, Williams NM, Williams J, Jones LA, Cardno AG, Murphy KC, Spurlock G, Riley B, Scambler P, Asherson P, McGuffin P, Owen MJ: No evidence for allelic association between schizophrenia and a polymorphism determining high or low catechol O-methyltransferase activity. Am J Psychiatry 1996; 153: 268-270

55. Palmatier MA, Kang AM, Kidd KK: Global variation in the frequencies of functionally different catechol-O-methyltransferase alleles. Biol Psychiatry 1999; 15:557-567

56. Bookheimer SY, Strojwas MH, Cohen MS, Saunders AM, PericakVance MA, Mazziotta JC, Small GW: Patterns of brain activation in people at risk for Alzheimer's disease. N Engl J Med 2000; 343:450-456

57. Barkley RA, Grodzinsky G, DuPaul G: Frontal lobe functions in attention deficit disorder with and without hyperactivity: a review and research report. J Abnorm Child Psychol 1992; 20: 163-188
58. Pennington BF, Ozonoff S: Executive functions and developmental psychopathology. J Child Psychol Psychiatry 1996; 37: 51-87

59. Cox CS: Neuropsychological abnormalities in obsessive-compulsive disorder and their assessments. Int Rev Psychiatry 1997; 9:45-60

60. Lyvers M: "Loss of control" in alcoholism and drug addiction: a neuroscientific interpretation. Exp Clin Psychopharmacol 2000; 8:225-249

61. Perlstein WM, Carter CS, Noll DC, Cohen JD: Relation of prefrontal cortex dysfunction to working memory and symptoms in schizophrenia. Am J Psychiatry 2001; 158:1105-1113

62. Weinberger DR, Egan MF, Bertolino A, Callicott JH, Mattay VS, Lipska BK, Berman KF, Goldberg TE: Prefrontal neurons and the genetics of schizophrenia. Biol Psychiatry 2001; 50:825-844

63. Pelosi L, Slade T, Blumhardt LD, Sharma VK: Working memory dysfunction in major depression: an event-related potential study. Clin Neurophysiol 2000; 111:1531-1543 